\title{
Coronary Artery Anomalies and Variations Detected In Computed Tomography Angiography
}

\author{
Ali Mahir Gündüz \\ Department of Radiology, Van Yuzuncu Yil University Medical Faculty, Van, Turkey
}

\begin{abstract}
There are many methods in the diagnosis of coronary artery diseases, and the reference method is catheter angiography. Coronary computed tomography angiography (c-CTA) is more valuable in the diagnosis of coronary artery and cardiac anomalies, plaques in the coronary artery wall, aortic dissection, myocardial bridges, osteal pathologies and coronary fistulas. In this study, we investigated the diversity and frequency of coronary artery anomalies and variations in c-CTA examinations performed in our unit.

We included 700 patients who underwent c-CTA in the study. The c-CTA images and reports of the patients were retrospectively evaluated, and the diversity and frequency of coronary artery anomalies and variations were investigated. The axial images, multiplanar reconstruction, maximum intensity projection and 3D volume rendering images were examined together and coronary artery stenosis, anomalies and variations were evaluated.

We detected intermediate artery in 126 patients and myocardial bridging in 87 patients. LMCA was not observed in 11 patients. Single coronary artery was observed in 3 patients. RCA showed an interarterial course between aorta and pulmonary artery in 1 patient.

C-CTA is an effective method in detecting coronary artery diseases, variations and anomalies. There are studies indicating that cCTA is superior to catheter angiography in the diagnosis of coronary artery anomalies. Coronary artery anomalies are the uncommon category of cardiac diseases. Their incidence in the community is $1-2 \%$. Our study supports that c-CTA is an imaging method that is used in the diagnosis of coronary artery anomalies and variations and provides detailed information.
\end{abstract}

Key Words: Computed tomography angiography, coronary artery, anomaly

\section{Introduction}

There are many methods in the diagnosis of coronary artery diseases, and the reference method is catheter angiography. Coronary computed tomography angiography (c-CTA) is more valuable in the diagnosis of coronary artery and cardiac anomalies, plaques in the coronary artery wall, aortic dissection, myocardial bridges, osteal pathologies and coronary fistulas $(1,2)$. Approximately $1 / 5$ of coronary artery anomalies may lead to serious symptoms such as arrhythmia, syncope, myocardial infarction or sudden death (3). For these reasons, it was aimed to investigate the diversity and frequency of coronary artery anomalies and variations in c-CTA examinations performed in our unit.

\section{Material and Methods}

The study was initiated after receiving approval from Van Yüzüncü Yıl University Ethics Committee.

Patient Selection: 700 patients who underwent cCTA in Van Yüzüncü Yll University, Faculty of Medicine, Department of Radiology between November 2018 and May 2019 were included in the study. The c-CTA images and reports of the patients were retrospectively evaluated, and the diversity and frequency of coronary artery anomalies and variations were investigated. 30 patients with poor image quality were not included in the study.

Coronary Computed Tomography Angiography Protocol: C-CTA examinations were performed with 128 slice CT [Siemens Definition AS +(Plus) device. The images were obtained by retrospective triggered ECG gating method. The patients with a heart rate above 75 were given $\beta$-adrenergic receptor blockers (50 mg metoprolol) approximately 1 hour before cCTA. $80-90 \mathrm{ml}$ of non-ionic contrast agent was delivered at a rate of $5-6 \mathrm{ml} / \mathrm{sec}$ from the right antecubital vein with an 18-20 Gauge needle using a double-headed automatic syringe. Shooting was automatically started by placing ROI (region of interest) to the left atrium by automatic bolus triggering technique.

Image Analysis: All evaluations were performed at a special workstation (Siemens Syngovia). In routine evaluation, heart rate, imaging quality, calcium scorepercentile value, coronary arteries-coronary dominance, main vessels and cardiac cavities in all patients were evaluated. Coronary artery dominance 


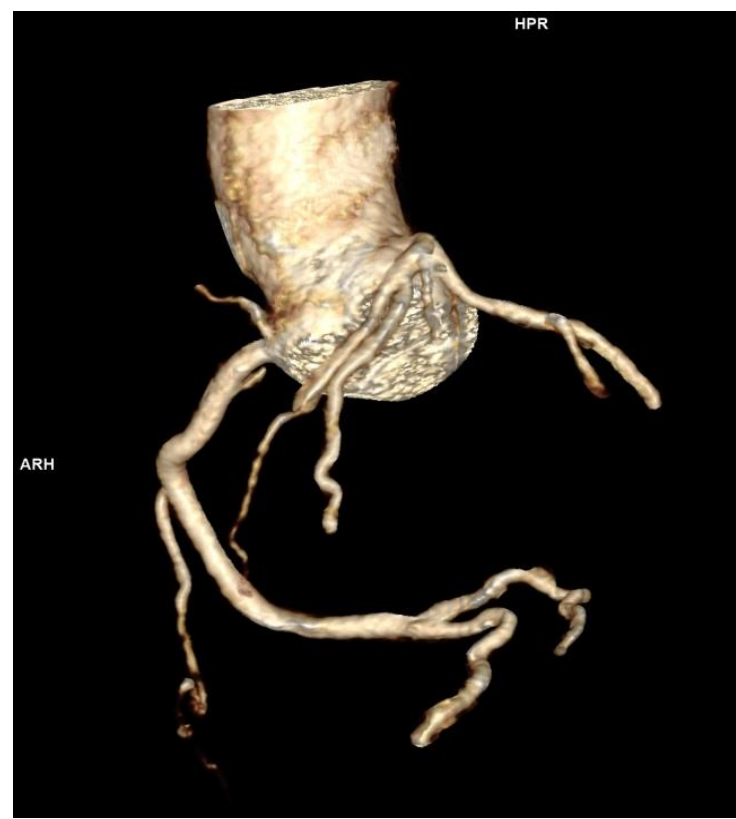

Fig. 1. RCA and conus artery originated directly and separately from the RSV in the volumetric CT images

was determined as follows: Right dominant circulation was present if the posterior descending artery (PDA) and posterolateral branch (PLB) originated from the right coronary artery (RCA). There was a left dominant circulation if PDA and PLB originated from the left circumflex artery ( $\mathrm{LCx}$ ). There was codominance if PDA originated from RCA and PLD originated from LCx. The axial images, multiplanar reconstruction (MPR), maximum intensity projection (MIP) and 3D volume rendering (VR) images were examined together and coronary artery stenosis, anomalies and variations were evaluated. Coronary artery anomalies and variations detected in all patients were noted separately from other findings.

\section{Results}

Our study included a total of 700 patients consisting of 380 males and 320 females aged between 21 and 75 years (average age 55 years). No anomaly, variation or pathology was observed in the coronary arteries of 273 of 700 patients $(39 \%)$. Coronary artery dominance was as follows; 67 patients $(9.57 \%)$ had left dominant circulation, 17 patients $(2.43 \%)$ had codominance, and the remaining patients $(88 \%)$ had right dominant circulation. The conus artery originated from RCA proximal in 407 patients $(58.14 \%)$ and from right sinus valsalva (RSV) or directly from the aorta in the remaining patients $(41.86 \%)$ (Figure 1). Sinoatrial nodal artery was observed as the second branch of RCA in 455

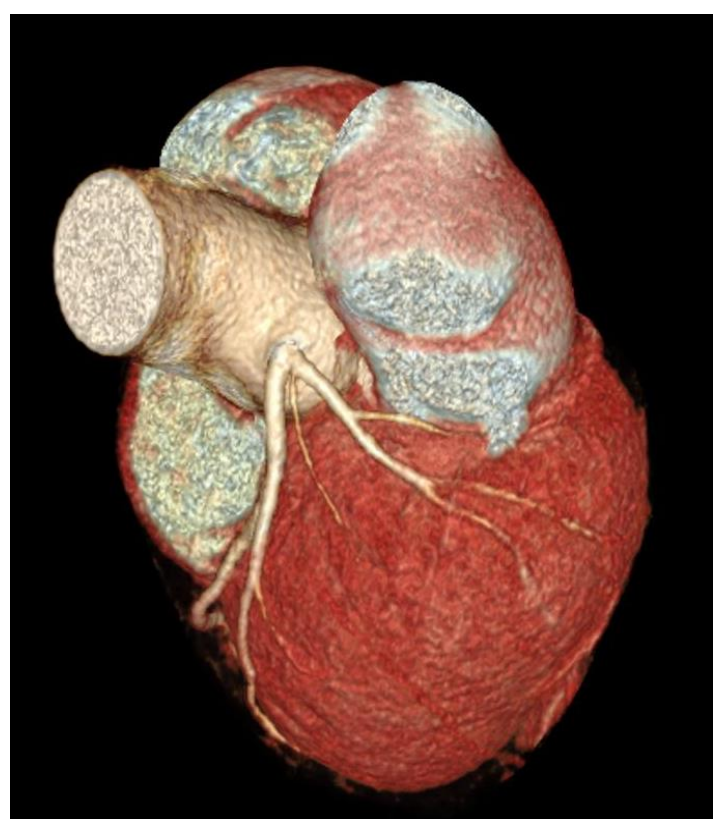

Fig. 2. Intermediate artery originating from between LAD and LCx appear in the volumetric CT images

patients $(65 \%)$.

The variation of the intermediate artery in the distal left main coronary artery (LMCA), between LAD (left anterior descending artery) and LCx was observed in 126 patients (18\%) (Figure 2). Myocardial bridging was observed in 87 patients $(12.43 \%)$, and 3 of these patients were observed in RCA, 3 of them were observed in LCx and the remaining of them were observed in LAD (Figure 3). LMCA was not observed in 11 patients (1.6\%), and LAD and LCx were found to be separated from the left sinus valsalva (LSV) in these patients (Figure 4). Single coronary artery was observed in 2 patients and LMCA originated from RCA in these patients (Figure 5). LCX originated from the RCA in 3 patients.

RCA showed an interarterial course between aorta and pulmonary artery in 1 patient (Figure 6). Coronary artery anomalies and variations found in our study are presented in Table 1.

Some important results that we excluded from the study but detected in routine screening were as the following: Stenosis leading to occlusion due to calcific-noncalcific plaques in coronary arteries were remarkable in 197 patients (28.1\%). 68 coronary artery stents were observed in 44 patients (6.3\%), and 14 of these stents were occluded. 8 of a total of 22 bypass grafts observed in 10 patients (1.4\%) had an occluded appearance. The patients with occluded stent and graft were referred to cardiology outpatient clinic.

It was observed that 2 patients had anomalous pulmonary venous return, 13 patients had atrial septal 

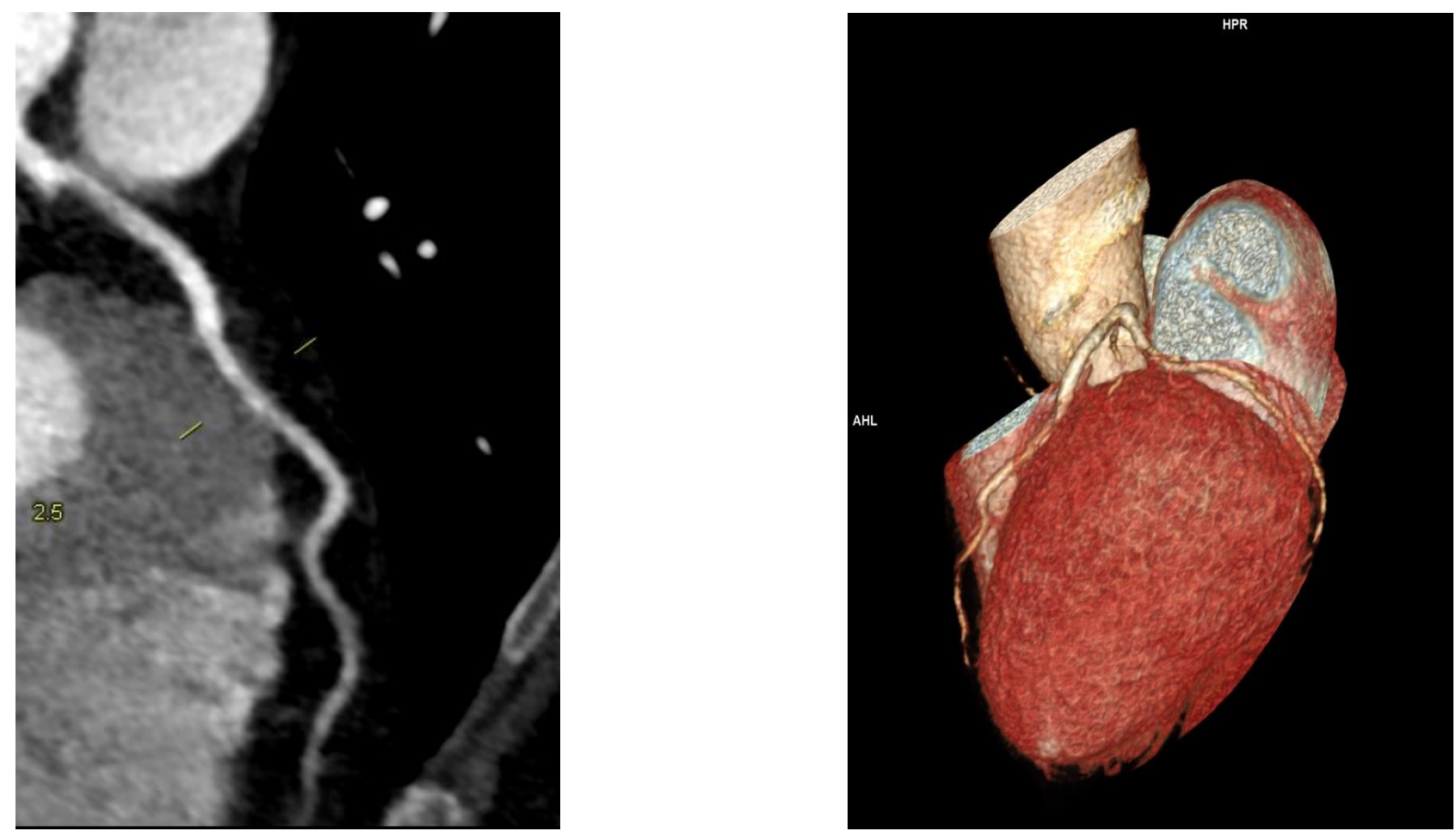

Fig. 3. Myocardial bridging in the proximal segment of LAD appear in the MPR (a) and volumetric CT (b) images
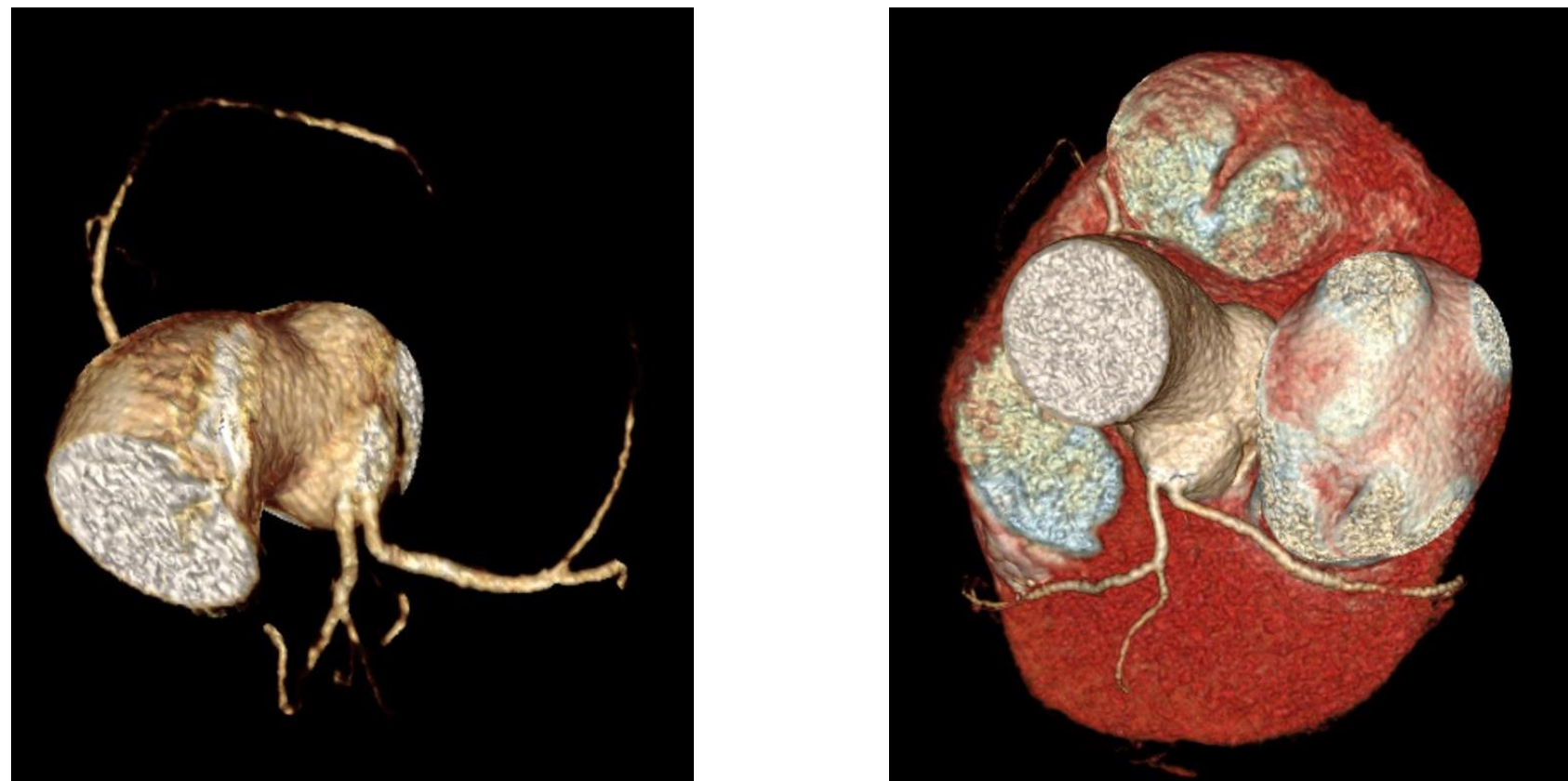

Fig. 4. LMCA is not observed in the volumetric CT images (a,b). LAD and LCx originate separately from the LSV

defect (ASD), 1 patient had tetralogy of fallot, 1 patient had tricuspid atresia, 1 patient had hypoplastic right heart, 1 patient had right aortic arch, and 1 patient had constrictive pericarditis.

\section{Discussion}

Catheter angiography is the gold standard in the diagnosis of coronary artery diseases. It was observed that coronary artery pathology was not detected in $1 / 4$ of the patients who underwent coronary catheter angiography, and there was a need for interventional procedures only in $1 / 3$ of the patients with pathology $(4,5)$. C-CTA is an effective method in detecting coronary artery diseases, variations and anomalies. There are studies indicating that c-CTA is superior to catheter angiography in the diagnosis of coronary artery anomalies. In a study with 1758 patients who

East J Med Volume:24, Number:4, October-December/2019 

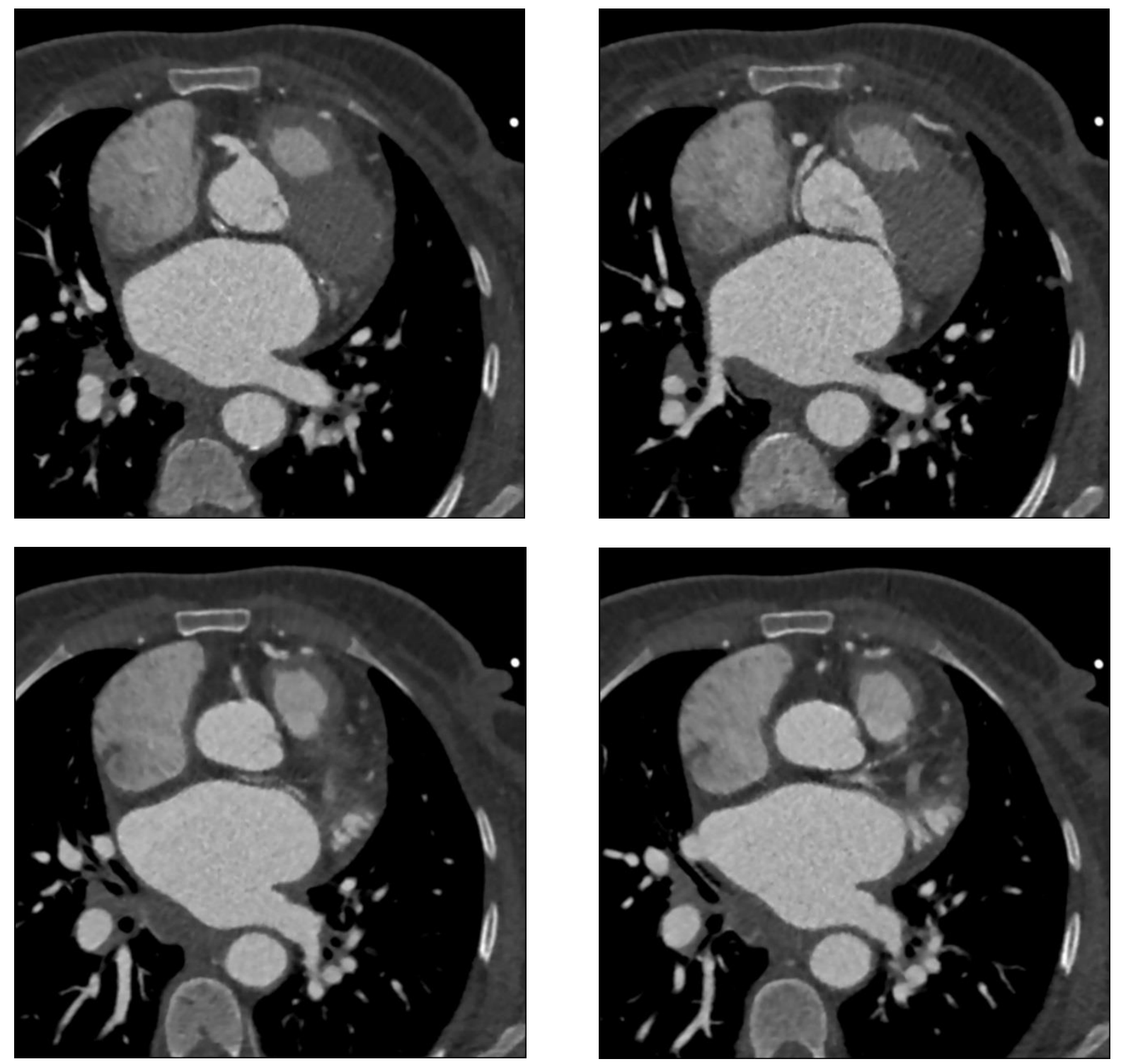

Fig. 5. LMCA originated from RCA in a patient with single coronary artery in the axial CT images
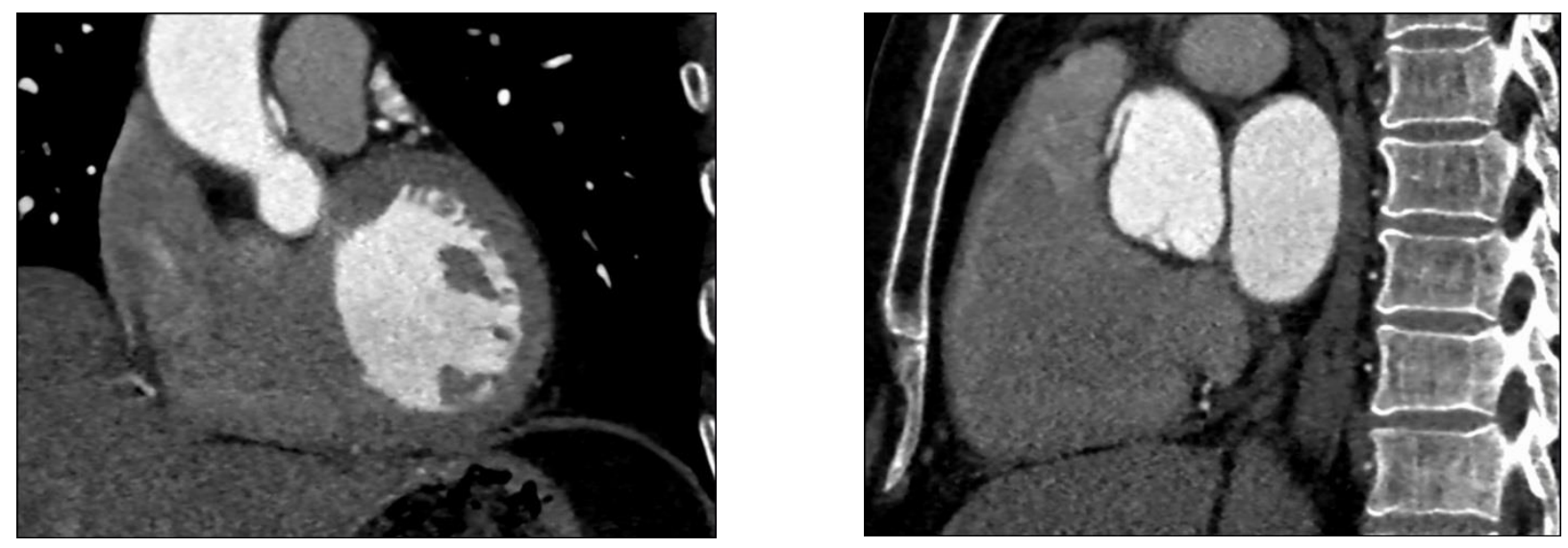

Fig. 6. RCA originating from the RSV and showing interarterial course appears in the reformate CT images

underwent c-CTA, coronary artery anomaly was detected in 28 patients. When catheter angiography was performed in 20 of these patients, anomaly could be detected only in 11 of them (6). Similar results were also obtained in different studies carried out by Komatsu and Shi $(7,8)$. 
Table 1. Coronary artery anomalies and variations detected in our study

\begin{tabular}{lcc}
\hline Anomalies and variations & Number & Percent $(\%)$ \\
\hline Right dominant circulation & 616 & 88 \\
Left dominant circulation & 67 & 9.57 \\
Codominance & 17 & 2.43 \\
Intermediate artery & 126 & 18 \\
Myocardial bridging & 88 & 12.43 \\
Absence of LMCA & 11 & 1.46 \\
Single coronary artery & 2 & 0.29 \\
LMCA originates from the RCA & 2 & 0.29 \\
LCX originating from RCA & 3 & 0.43 \\
Interarterial course & 1 & 0.14 \\
\hline
\end{tabular}

Coronary artery anomalies are the uncommon category of cardiac diseases. Their incidence in the community is $1-2 \%$ (9). In the largest angiography series consisting of 126595 patients, Yamanaka and Hobbs reported that the incidence of abnormal coronary artery was $1.3 \%$ (10). Srinivasan et al. evaluated 1495 patients who underwent c-CTA in the evaluation of coronary artery disease, and they found the incidence of anomaly as $0.8 \%$ (11).

However, the frequency of anomaly increases if it is included in coronary artery anomalies in myocardial bridging (12). They are detected incidentally since they are usually asymptomatic.

Early diagnosis is important because of their potential to cause myocardial ischemia and sudden death. In young adults, approximately half of nontraumatic sudden deaths are caused by cardiac anomalies. The underlying reason in $2 / 3$ of them is the coronary artery anomaly (13).

In our study consisting of 700 patients who underwent c-CTA, no anomaly, variation or pathology in coronary arteries was observed in 273 patients (39\%), and coronary catheter angiography was not needed. The main variations and anomalies we detected were coronary artery origin and course anomalies, intermediate artery and myocardial bridging.

The length of LMCA (left main coronary artery) is between $0.5-2 \mathrm{~cm}$ and is divided into two main branches: LAD (left anterior descending artery) and LCx (left circumflex artery). Ramus intermedius artery originating from between LAD and LCx in distal to LMCA is one of the most common variations, and its incidence was reported up to $33 \%(14,15)$. In our study, we detected intermediate artery variation in 126 patients $(18 \%)$.

We detected myocardial bridging in a total of 87 patients $(12.43 \%)$ including RCA (right coronary artery) in 3 of them, LCx in 3 of them, and LAD in the rest of them. Myocardial bridging is the course of coronary arteries within the myocardium. In a study carried out with c-CTA, its prevalence in our population was reported to be $3.5 \%$. It is most commonly observed in LAD. It is usually asymptomatic and may be observed with chest pain, arrhythmia, infarct or sudden death (16).

We detected coronary origin anomaly in 17 patients $(2.4 \%)$. While LMCA was not observed in 11 of them $(1.6 \%)$, LAD and LCx originated directly and separately from the LSV. LCX originated from the RCA in 3 patients. LMCA originated from RCA in 2 patients with single coronary artery. In single coronary artery, the arteries that supply the heart with blood originate from a single site. They are rare and their incidence is $0.024-0.044 \%$ (17).

RCA had an interarterial progression in 1 patient $(0.14 \%)$. Interarterial coronary artery is localized between aorta and pulmonary artery $(7,1)$. It is the most serious coronary artery anomaly and is defined as malignant, and sudden cardiac death may occur (19).

One of the limitations of our study is that no comparison was performed with coronary catheter angiography. Furthermore, our study did not include healthy individuals, it was carried out on the population presenting to the hospital due to any symptom.

Our study supports that c-CTA is an imaging method that is used in the diagnosis of coronary artery anomalies and variations and provides detailed information. The advantages of c-CTA are that it is easily applicable and a fast and minimally invasive modality.

\section{References}

1. Kantarc1 M, Doğanay S, Karçaaltıncaba M, et al. Clinical situations in which coronary CT 
angiography confers superior diagnostic information compared with coronary angiography. Diagn Interv Radiol 2012; 18: 261-269.

2. Canyiğit M, Hazırolan T, Karçaaltıncaba M, et al. Myocardial bridging as evaluated by 16 row MDCT. Eur J Radiol 2009; 69: 156-164.

3. Datta J, White CS, Gilkeson RC, et al. Anomalous coronary arteries in adults: Depiction at multidetector row CT angiography. Radiology 2005; 235: 812-818.

4. Papaconstantinou HD, Marshall EJ, Burrell CJ. Diagnostic cardiac catheterzation in a hospital without on site cardiac surgery. Heart 1999; 81: 465-469.

5. Hazırolan T. Koroner arterlerin çok dedektörlü bilgisayarlı tomografi ile görüntülenmesi. Hacettepe Tip Dergisi 2006; 37: 6-13.

6. Schmitt R, Froehner S, Brunn J, et al. Congenital anomalies of the coronary arteries: imaging with contrast-enhanced, multidetector computed tomography. Eur Radiol 2005; 15: 1110-1121.

7. Komatsu S, Sato Y, Ichikawa M, et al. Anomalous coronary arteries in adults detected by multislice computed tomography: presentation of cases from multicenter registry and review of the literature. Heart Vessels 2008; 23: 26-34.

8. Shi H, Aschoff AJ, Brambs HJ, Hoffmann MH. Multislice CT imaging of anomalous coronary arteries. Eur Radiol 2004; 14: 2172-2181.

9. Fujimoto S, Kondo T, Orihara $\mathrm{T}$, et al. Prevalence of anomalous origin of coronary artery detected by multi-detector computed tomography at one center. J Cardiol 2011; 57: 69-76.

10. Yamanaka O, Hobbs RE. Coronary artery anomalies in 126,595 patients undergoing coronary arteriography. Cathet Cardiovasc Diagn 1990; 21: 28-40.
11. Srinivasan KG, Gaikwad A, Kannan BR, Ritesh K, Ushanandini KP. Congenital coronary artery anomalies: diagnosis with 64 slice multidetector row computed tomography coronary angiography: a single-centre study. J Med Imaging Radiat Oncol 2008; 52: 148-154.

12. Cademartiri F, La Grutta L, Malagò R, et al. Prevalence of anatomical variants and coronary anomalies in 543 consecutive patients studied with 64-slice CT coronary angiography. Eur Radiol 2008; 18: 781-791.

13. Öztürk E. Coronary artery variations. Türkiye Klinikleri J Radiol-Special Topics 2011; 4: 1318.

14. Koşar P, Ergun E, Cansu Ö, Koşar U. Anatomic variation and anomalies of the coronary arteries: 64CT angiographic appearance. Diagn Interv Radiol 2009; 15: 275 283.

15. Dewey M, Kroft LJM.Anatomy. In Dewey M, ed. Coronary CT angiography. Berlin, Springer 2009; 11-26.

16. Kantarc1 M, Duran C, Durur I, et al. Detection of myocardial bridging with ECG-gated MDCT and multiplanar reconstruction. ACR Am J Roentgenol 2006; 186: 391-394.

17. Zeina AR, Blinder J, Sharif D, Rosenschein U, Barmeir E. Congenital coronary artery anomalies in adults: non-invasive assessment with multidetector CT. Br J Radiol 2009; 82: 254-261.

18. Shriki JE, Shinbane JS, Rashid MA, et al. Identifying, characterizing, and classifying congenital anomalies of the coronary arteries. Radiographics 2012; 32: 453-468.

19. Duran C, Kantarc1 M, Durur Subaşı İ, et al. Remarkable anatomic anomalies of coronary arteries and their clinical importance: a multidedector computed tomography angiographic study. J Comput Assist Tomogr 2006; 30: 939-948. 